\title{
Preparing a Successful Scientific Abstract for Professional Meetings in the Data Sciences
}

By Richard F. Ittenbach, PhD, PSTAT

Alexander C. Bragat, PMP

Susan K. Howard, MSN

Abstracts constitute an important part of the scientific process. They allow readers to assess important attributes of a given project or study in a quick and efficient manner. Each year the Society of Clinical Data Management (SCDM), similar to other professional societies, issues a 'Call for Abstracts' in which data professionals are encouraged to submit summaries of their work for possible presentation at the annual meeting. If an abstract is accepted, authors will be asked to share their work at the meeting through a podium, poster, panel, or round-table presentation. The purpose of this article is to educate SCDM members about the fundamentals of writing an abstract, including its overarching purpose, key components, and potential for contributions to both the profession and science, more generally. Recommendations for writing abstracts are also provided.

\section{Purpose}

The primary purpose of a scientific abstract is to succinctly describe the objectives, methods used, and relevant findings of a given study. In most but certainly not all cases, a given study has been completed and all results are known at that the time an abstract is composed. Most importantly, the study's authors will know whether or not their study objectives were achieved-findings that serve as the basis for an abstract submission.

\section{Key Components}

While there is no universally agreed upon criteria for the perfect abstract, there is consistency as to the general types of information that should be included'. For purposes of SCDM abstracts, potential authors are encouraged to include the following five sections in their abstract submissions.

- Background Summary statement of the science to date, including key findings and gaps in the professional literature.

All information presented should be based upon the scientific literature as opposed to

speculation or personal perspectives or preferences.

- Objectives Organizing framework for the study. Acceptable forms of objectives include hypotheses, research questions, or specific aims.

- Methods Description of subjects, clinical or operational procedures, and analytical methods used.
- Results

Findings from the analytic phase. The findings may take many forms, including quantitative or qualitative, descriptive or inferential. All findings can be valuable if analyzed with rigor and within the context of the scientific method.

- Conclusion Summary statements pertaining to each objective and recommendations for practice or additional inquiry.

Within the biomedical sciences, two types of abstracts are most often used: structured and unstructured. In the case of structured abstracts, which are often considered to be more complete and easier to read', each of the aforementioned sections consists of a distinct 2 to 3 sentence paragraph. In unstructured abstracts, the same elements are required, just within a single uninterrupted paragraph. See Appendices A and B for a hypothetical example of each. Importantly, not all societies request the same information in the same way for a given abstract, and requirements may vary from year to year. Word limits are generally strict and typically do not exceed 250 words. For these reasons, care should be taken to adhere to the guidelines closely when composing an abstract for a particular meeting.

\section{Contributions to Science and the Profession}

Although abstracts are small in size, their contributions to science can be far-reaching. Most importantly, abstracts provide a mechanism through which researchers can communicate with others-the components of a given study and the rigor with which it was conducted. Most often abstracts serve as introductions to a publication. Less well known is that abstracts are used by informationists when indexing a study for scientific databases (e.g., PubMed, SCOPUS, ISI Web of Science). It is through these databases that others who may not have immediate access to a particular publication or presentation become aware of it. In short, abstracts serve as both a communication and quality assurance tools that researchers have conducted their studies in accord with established scientific principles.

When it comes to conference presentations, specifically, abstracts have a heightened sense of importance. According to von Elm et al., as few as one-third of abstracts submitted for presentation at biomedical science meetings may make it in a complete form to publication ${ }^{2}$. For those studies that do not make it to publication, an abstract may be the only written record of a team's scientific work ${ }^{2,3}$. A well-written abstract may mean the difference between getting on the program or not. For others, it may mean the difference between a podium and a poster or roundtable presentation. 
To this extent, potential presenters who spend time composing their abstracts in a rigorous and thoughtful manner are likely to produce better, more meaningful and more informative abstracts than those who do not.

Presenters often believe that their study or project can only be presented in a certain format-but that is rarely the case. While some material may be more conducive to a certain type of presentation (e.g., podium presentation or educational workshop), the reality is that most material can be presented in many different ways with equal integrity. Practically speaking, a meeting's planning committee may have only so many openings for a given type of presentation, so potential authors should consider writing their abstract such that the material can be presented in multiple ways. Willingness to have one's work considered for different types of presentations is an important but practical means of being able to share one's work professionally with others.

Conference abstracts often have a flexibility that other types of abstracts do not, particularly when it comes to conceptual or process related work. Many professional societies such as SCDM strive to introduce their members to emerging lines of research and practice-to keep them up-to-date on new methods, practices, and technologies. Very often that includes innovative types of research for which the field has yet to reach consensus. When this is the case, the new mechanisms (or methods) should be clearly stated so they are not confused with completed or previously validated findings. The reporting of these methods should be no less rigorous even if the empirical work is still developing. Whatever the lines of inquiry are, the limitations and criteria for evaluation need to be clearly stated.

In the case of conceptual or more process related studies (e.g., quality assurance methods, improved feedback cycles), care should be taken to highlight characteristics of the specific processes used along with criteria as to how these processes were judged to be successful. Without clear and objective end points, it may be difficult for others to know with certainty that the new method or process was indeed better than the standard. Investigators should also include the perceived strengths and weaknesses of the new processes along with any limitations to implementation. Putting these methods in a scientific context, with objective standards or thresholds shifts the discussion away from personal preferences and testimonials toward objective and reproducible forms of evaluation.

Composing an abstract for a professional meeting must be premised on science but rely on fundamentals of sound communication and potential for widespread appeal. Conference planning committees may receive hundreds or even thousands of abstracts to review in a short period of time. Clearly articulated, informative, interesting studies and projects go a long way toward getting reviewers' attention and an invitation to present at a professional meeting. Readers may wish to consult Cook and Bordage's "Twelve tips on writing abstracts and titles: How to get people to use and cite your work" 4 (2016) for suggestions for ways in which authors can get their abstracts noticed and thereby increase the likelihood that their papers will be considered useful by others.

\section{Recommendations}

There are many routes to preparing a successful abstract for a scientific meeting within the data sciences. The most important consideration is to have a research product or process that is not only scientifically sound and that you are proud of, but that you believe others would want to know about. The second consideration then is to organize your findings in a scientifically acceptable manner using the five steps noted above. Scientific abstracts follow a conventional structure that has been adopted across disciplines for decades. Deviating from the established structure can be done, but is not without its risks. For purposes of submitting to SCDM or other professional data science meetings, we offer the following general recommendations that may prove helpful:

(1) Identify the most important points of your study within each of the five aforementioned areas and state them clearly in your abstract. Strive to be thorough but not excessive.

(2) Write the abstract in a clear and consistent form with proper spelling, grammar and syntax. It is generally recommended that abstracts be written in a passive voice; however, there are times when an active voice is needed and/or preferred.

(3) Adhere closely to the guidelines for the particular scientific meeting. Read, re-read and re-read again the guidelines for submission so that your abstract meets both the technical and nontechnical requirements of the conference planning committee.

(4) It should be immediately clear what the objectives of the study or project are and whether or not they were supported by the data. In the case of more process-oriented abstracts, articulating what the novel processes are and offering evidence as to why they are considered to be superior to the more traditional ones is crucial.

(5) Write the abstract in such a way that if it is not accepted for one type of presentation (e.g., podium), it could be considered for another (e.g., poster). Planning committees appreciate abstracts that are flexible enough that they can be positioned in different places in a program.

\section{References}

1. James Hartley, "Current findings from research on structured abstracts: An update," Journal of the Medical Library Association 102, no. 3 (2014): 146-148.

2. Chittaranjan Andrade, "How to write a good abstract for a scientific paper or conference presentation," Indian Journal of Psychiatry 53, no. 2 (2011): 172-175.

3. Erik von Elm, Michael C. Constanza, Bernhard Walder, and Martin R. Tramer, "More insight into the fate of biomedical meeting abstracts: A systematic review," BMC Medical Research Methodology 3, no. 12 (2003): 12, accessed December 27, 2016, http://bmcmedresmethodol. biomedcentral.com/articles/10.1186/1471-2288-3-12

4. David A. Cook and George Bordage, "Twelve tips on writing abstracts and titles: How to get people to use and cite your work," Medical Teacher 38, no. 11 (2016): 1100-1104. 


\section{Appendix A}

Example of a Hypothetical Structured Abstract

\section{Title: Biomarker Endpoints in Investigator-Initiated Trials are Associated with} Study Objective Success

Background: Well-validated biomarker assays used in early Phase I and II studies are often used as proof of concept studies when providing preliminary safety and efficacy evidence for new therapeutic interventions. In Phase III studies, biomarkers are often used as surrogate endpoints for other outcomes. Investigator-initiated trials (IIT) offer an alternative but minimally used mechanism for inclusion of biomarker assays in innovative oncology studies.

Objective: To assess and compare the success rates of IITs using biomarkers as primary endpoints with the success rates of IITs using biomarkers as secondary endpoints.

Methods: IIT studies reporting results in clinicaltrials.gov were compiled and categorized according to their use of biomarker endpoints or other comparable measures. Success rates were computed for IIT studies for which both primary and secondary endpoints, individually, and compared using a two-sample test of proportion.

Results: Results from 200 published studies ( $n=100 /$ group) with and without biomarker endpoints were available for analyses. In the biomarker group, $30 \%$ of studies met their objectives while $70 \%$ either did not meet their objective or were inconclusive. In the non-biomarker group, 19\% of studies met their objectives while $81 \%$ either did not meet their objective or were inclusive $(p=0.07)$.

Conclusion: Despite the appearance of a $11 \%$ difference in success rates favoring biomarker studies meeting their objectives, the difference was not statistically significant $(p=0.07)$. More research is needed to estimate the stability of this finding in subsequent trials.

\section{Appendix B}

Example of a Hypothetical Unstructured Abstract

\section{Biomarker Endpoints in Investigator-Initiated Trials are Associated with Study} Objective Success

Well-validated biomarker assays used in early Phase I and II studies are often used as proof of conceptstudies when providing preliminary safety and efficacy evidence for new therapeutic interventions. In Phase III studies, biomarkers are often used as surrogate endpoints for other outcomes. Investigator-initiated trials (IIT) offer an alternative but minimally used mechanism for inclusion of biomarker assays in innovative oncology studies. This report aims to assess and compare the success rates of IITs using biomarkers as primary endpoints with the success rates of IITs using biomarkers as secondary endpoints. IIT studies reporting results in clinicaltrials.gov were compiled and categorized according to their use of biomarker endpoints or other comparable measures. Success rates were computed for IIT studies for which both primary and secondary endpoints, individually, and compared using a two-sample test of proportion. Results from 200 published studies ( $n=100 /$ group) with and without biomarker endpoints were available for analyses. In the biomarker group, $30 \%$ of studies met their objectives while $70 \%$ either did not meet their objective or were inconclusive. In the non-biomarker group, 19\% of studies met their objectives while $81 \%$ either did not meet their objective or were inclusive ( $p=0.07)$. Despite the appearance of a $11 \%$ difference in success rates favoring biomarker studies meeting their objectives, the difference was not statistically significant $(p=0.07)$. More research is needed to estimate the stability of this finding in subsequent trials. 